A.H. GLENN AND ASSOCIATES
ALFRED H. GLENN
Certified Consulting Meteorologist
Consultants in Meteorology and Oceanography since 1946•
Professional Engineer
New Orleans Lakefront Airport
P. O. Box 26337
New Orleans, LA 70126

\section{WEATHER SERVICES CORPORATION} PETER R. LEAVITT

JOHN P. MURPHY

\section{Certified Consulting Meteorologists}

Worldwide operational meteorology and consulting, 24 hours per day, 7 days per week; serving industry, media, and government.

420 Bedford St.

Lexington, MA 02173

Tel: $617-676-1000$

Fax: 617-676-1001 e-mail: info@wx.com

\section{NORTH AMERICAN WEATHER}

\section{CONSULTANTS A TRC COMPANY}

GEORGE W. WILKERSON, MANAGER, AIR QUALITY SERVICES

\section{Certified Consulting Meteorologist}

Weather Modification • Air Quality Surveys \& Field Studies • Applied Research • Forensic Meteorology

AEROMET, INC.

D. RAY BOOKER, PH.D., PRESIDENT

\section{Certified Consulting Meteorologist}

Instrumented Aircraft • Aerospace Meteorology $\bullet R \& D \cdot$ Forecasts

P.O. Box 701767

Tulsa, OK 74170-1767

$918-299-2621$

\section{RICHARD E. CALE}

\section{Certified Consulting Meteorologist}

Environmental Research Applications • Aviation Weather Investigations • Planning Services

\section{MURRAY AND TRETTEL, INCORPORATED}

JOHN R. MURRAY

JOHN P. BRADLEY

DENNIS W. TRETTEL

\section{Certified Consulting Meteorologists}

Founded 1946, Micro/Operational Forecasting • Media • Air Quality/ Meteorological/PSD Monitoring/Studies • Forensic Research • Dispersion Modeling $\bullet$ Nuclear Emergency Support

414 W. Frontage Rd.

Northfield, IL 60093

(708)446-7800/FAX (708)446-1420

(312) $273-5600$

\section{SURFACE SYSTEMS, INC.}

\section{ARMAND R. IACCHEO}

\section{Certified Consulting Meteorologist}

SPECIALIZING IN: Road and runway weather information systems • Operational forecasting and consulting for the utility and transportation industries

11612 Lilburn Park Road

314-569-1002

St. Louis, MO 63146 FAX 314-569-3567

\section{CLIMATOLOGICAL CONSULTING CORPORATION}

WALTER A. BOHAN

\section{Certified Consulting Meteorologist}

Specializing in Forensic Meteorology
P.O. Box 736

Park Ridge, IL 60068

708-825-3677
150 Shope Creek Road Asheville, NC 28805 704-298-4237

\section{METRO MONITORING SERVICES \\ ERWIN K. KAUPER}

\section{Certified Consulting Meteorologist}

Environmental Impact \& Site Surveys • Air Pollution Field Studies • Diffusion Analysis • Weather \& Air Quality Forecasting • Air Pollution Monitoring Instrumentation

P.O. Box 4584

Covina, CA 91723

818-332-8411

\section{WEATHER RESEARCH CENTER}

JOHN C. FREEMAN, DIRECTOR OF RESEARCH JILL F. HASLING, DIRECTOR OF OPERATIONS

\section{Certified Consulting Meteorologists}

Worldwide Weather \& Oceanographic Forecasting • Climatology • Expert Testimony $\bullet$ Research in Meteorology \& Oceanography ${ }^{\bullet}$ Wave Spectra • Software Development • Observing Network Design 


\section{SPACE RESERVED FOR CERTIFIED CONSULTING METEOROLOGISTS}

\section{W. BOYNTON BECKWITH}

\section{Certified Consulting Meteorologist}

Aviation-Accident Investigation \& Operational Applications • Mesometeorological Analyses • Climatological Studies • Expert Testimony

14728 Caminito Orense Oeste

San Diego, CA 92129

$619-672-1565$

\section{TRC ENVIRONMENTAL CORP.}

GALE F. HOFFNAGLE

LYSA G. MODICA

DOUGLAS R. MURRAY

\section{Certified Consulting Meteorologists}

Environmental Consulting \& Research • Applied Meteorology • Air Quality and Meteorological Monitoring • Diffusion Modeling • Tracer Studies • Air Toxics Monitoring • Expert Testimony

1-800-TRC-5601

Offices in major industrial centers throughout the United States

\section{APPLIED METEOROLOGY, INC.}

JOHN W. HATHORN

\section{Certified Consulting Meteorologist}

Meteorological Consulting \& Analysis • Air Quality Modeling \&

Monitoring • Site Selection \& Permitting $\bullet$ Environmental Data

Acquisition Systems \& Network with Remote-Control

9000 Southwest Freeway

Suite 400

Houston, TX 77074

713-777-0106

\section{INTERNATIONAL CENTER FOR THE SOLUTION OF ENVIRONMENTAL PROBLEMS \\ JOSEPH L. GOLDMAN, PH.D., TECHNICAL DIRECTOR}

\section{Certified Consulting Meteorologist}

Broad Scope Assessments by Interdisciplinary Experts Worldwide - Specialize in Mesometeorological Variations • Climate Change • Problems Posed, Solutions Implemented \& Demonstrated

\section{OCEANROUTES}

A WNI Weathernews Company

ROBERT L. COHEN

JAY P. GANZ

DONALD F. SCHUKRAFT

\section{Certified Consulting Meteorologists}

Consultants in Meteorology \& Oceanography • Optimum Ship

Routing • Site Specific Spectral Wave Forecasting • Specialized

Graphics • Oil Spill Modeling • Engineering-Design Site and Tow

Route Analyses - Environmental Monitoring \& Instrumentation

680 W. Maude Ave.

408-245-3600

Sunnyvale, CA $94086-3518$

FAX 408-245-5301
AEROVIRONMENT INC.

PAUL B. MACCREADY, JR., PH.D.

WILLIAM C. BRICK

KENNETH H. UNDERWOOD, PH.D.

\section{Certified Consulting Meteorologists}

Air Quality/Meteorology/Hazardous Waste Monitoring • Diffusion Modeling • Tracer Experiments • Instrumentation-Acoustic Radar, Air Samplers • Wind Energy Assessments/Wind Engineering

P.O. Box 5031

Monrovia, CA 91017-7131 Santa Barbara, CA 805-967-7699 818-357-9983 Denver, CO 303-989-2667

McVEHIL-MONNETT ASSOCIATES, INC. GEORGE E. McVEHIL, PH.D.

\section{Certified Consulting Meteorologist}

Air Quality Analysis and Monitoring • Permitting • Dispersion Modeling • Air Toxics • Meteorological Analysis • Industrial Meteorology $\bullet$ Litigation Support $\bullet$ Expert Testimony

44 Inverness Drive East

Building $\mathrm{C}$

303-790-1332

Englewood, CO 80112 FAX 303-790-7820

SIMPSON WEATHER ASSOCIATES, INC.

M. GARSTANG, PH.D.

G. D. EMMITT, PH.D.

R.H. SIMPSON, PH.D.

\section{Cerified Consulting Meteorologists}

Environmental Hazard/Risk Assessment • Air Quality ModelingMonitoring $\bullet$ Instrumentation - towers, lidars, surface network • Software Development • Environmental Simulations

809 E. Jefferson St.

804-979-3571

Charlottesville, VA 22902 FAX: 804-979-5599

\section{CLIMATOLOGICAL CONSULTING CORPORATION}

WILLIAM H. HAGGARD

DR. WERNER A. BAUM

STEPHEN W. SMUTZ

\section{Certified Consulting Meteorologists}

Specializing in Forensic Meteorology

150 Shope Creek Road

Asheville, NC 28805-9795

$704-298-4237$

DAMES \& MOORE

PERRY W. FISHER, PH.D.

JOHN A. FOSTER

JAMES W. LITTLE

\section{Certified Consulting Meteorologists}

Industrial Meteorology • Air Quality Modeling/Monitoring $\bullet P S D$

Permit Application Preparation - Expert Testimony • Climatological

Studies • Nuclear Meteorological Services and Analyses

Rolling Meadows, IL

Atlanta, GA

708-228-0707

404-262-2915

Certified Consulting Meteorologist: The certification program of the American Meteorological Society is aimed at fostering the establishment and maintenance of a high level of professional competency, and mature and ethical counsel, in the field of consulting meteorology. Requirements of knowledge, experience, and character are determined by a five-person board. Objectives of the program and application procedures are described in full detail in the August 1995 Bulletin (pp. 1416-1420). 


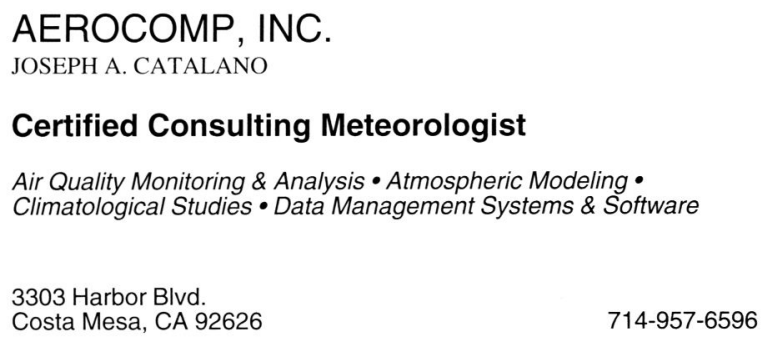

\section{Certified Consulting Meteorologist}

Air Quality Monitoring \& Analysis • Atmospheric Modeling • Climatological Studies • Data Management Systems \& Software

3303 Harbor Blvd.

Costa Mesa, CA 92626

$714-957-6596$

\section{AEROMATRIX INC. \\ CONRAD J. MASON, PH.D., PRESIDENT}

\section{Certified Consulting Meteorologist}

Solar Energy Surveys • Biometeorology • Meteorological \& Air Quality Monitoring $\bullet$ Dispersion Modeling $\bullet$ Site Surveys $\bullet$ Expert Testimony

3640 E. Huron River Dr.

Ann Arbor, Ml 48104

$313-971-2244$

\section{ATMOSPHERICS INCORPORATED}

THOMAS J. HENDERSON, PRESIDENT

\section{Certified Consulting Meteorologist}

Weather Modification Operation • Cloud Physics Research • Airborne Instrumentation - Weather Radar Applications • Aerosol Measurements • General Meteorological Services

\section{EDWIN X BERRY \& ASSOCIATES \\ EDWIN X BERRY, PH.D.}

\section{Certified Consulting Meteorologist}

Atmospheric Physics $\bullet$ Computer Models $\bullet$ Windows Programming Expert Testimony $\bullet$ People's Choice Award, 1993 Windows World Open 1994 Smithsonian Software Award Nomination

6040 Verner Avenue

916-344-7222

Sacramento, CA 95841-2032

FAX $916-344-7243$

\section{FREESE-NOTIS WEATHER, INC.}

HARVEY FREESE, M.S.

CHARLES NOTIS, M.S.

\section{Certified Consulting Meteorologists}

Worldwide Forecasts for: Agricultural \& Energy Commodities •

Construction $\bullet$ Highway Departments $\bullet$ Media $\bullet$ Forensic Meteorology - INTERNET SERVICES

2411 Grand Ave

Des Moines, IA 50312

$515-282-9310$

Fax: 515-282-6832

E-mail: hfreese@weather.net

Internet: http://www.weather.net/fn/ams
WEATHER CONSULTANTS

INCORPORATED

JOHN T. WALSER, PRESIDENT

\section{Certified Consulting Meteorologist}

Specializing in Weather Resources Management Design and Operations • Meteorological Observatory Operations • Forensic Meteorology

P.O. Box 3414

Santa Rosa, CA 95402-3414

Phone/Fax: 707-829-5370

\section{LYLE E. BROSCHÉ}

\section{Certified Consulting Meteorologist}

Specializing in Long-Range Weather Outlooks and Consulting

1347 Silver Lake Drive

Melbourne, FL 32940

407-259-1615

DOW ENVIRONMENTAL INC.

AMIRAM ROFFMAN, PH.D.

\section{Certified Consulting Meteorologist}

Air Quality Consulting, Diffusion Modeling, Air Toxics • Monitoring \& Field Programs • Environmental Studies, Hazardous \& Solid Waste Programs $\bullet$ Risk Assessment $\bullet$ Health \& Safety $\bullet$ Remediation

Penn Center West, Bldg. III, Suite 300

Pittsburgh, PA 15276

412-788-2717

Regional offices in Rockville, MD; Fairfield, NJ; Tampa, FL; Houston, TX; Salt Lake City, UT; San Franscico, CA; Los Angeles, CA

WILKENS WEATHER TECHNOLOGIES, INC. RICHARD B. WILKENS, EXECUTIVE VICE-PRESIDENT

\section{Certified Consulting Meteorologist}

Specialists in Marine, Aviation \& Industrial Forecasting Worldwide • Hindcast \& Climatological Studies • Computerized Flight Plans

\section{COURTNEY CONSULTANTS, INC.}

F.E. COURTNEY, PRESIDENT GEORGE W. REYNOLDS, PH.D

\section{Certified Consulting Meteorologists}

Meteorology, Modeling, Monitoring, Permits, Noise, Dust, Storms, Air Quality, Testimony, Accidents, Severe Weather

\section{Carriage Dr.}

Atlanta, GA 30328

404-256-2487 
PARSONS ENGINEERING SCIENCE, INC. DAVID B. SMITH ROBERT A. BAXTER ALFRED M. KLAUSMANN

\section{Certified Consulting Meteorologists}

Air Toxics/Air Quality/Meteorological Monitoring $\bullet$ Dispersion Modeling • Source Testing • Risk Assessment • Applied Climatology • Clean Air Act Consulting and Permitting

617-859-2000

Prudential Center

Boston, MA 02199

California, Ohio, Texas, and Virginia

\section{WEATHER APPLICATIONS}

DUANE R. (BOB) GOODMAN

\section{Certified Consulting Meteorologist}

Meteorology for Hazardous Waste Site Remediation, Climatology, Air Quality, Consulting, Forensic Meteorology

5812-314th St. Ct. S.

Roy, WA 98580

206-843-1374

\section{METEOROLOGICAL STANDARDS INSTITUTE}

THOMAS J. LOCKHART

\section{Certified Consulting Meteorologist}

Quality Assurance Plans and Audits $\bullet$ System Specifications • Training Workshops in Measurement and Quality Assurance • Instrument

Testing $\bullet$ Regulatory and Forensic Consulting

841 Seventh Lane, P.O. Box 26

Fox Island, WA 98333

206-549-2179

\section{EINAR L. HOVIND \\ Certified Consulting Meteorologist \\ Industrial Meteorology • Air Quality Analysis $\bullet$ Weather Modification \\ Services $\bullet$ Climatology $\bullet$ Forensic Meteorology}

4152 Primavera Rd.

Santa Barbara, CA 93110

805-964-8218

WEATHER NETWORK, INC.

A WNI Weathernews Company

DONALD F. SCHUKRAFT

\section{Certified Consulting Meteorologist}

Operational Weather Forecasts \& Climatological Studies • Instrumentation $\bullet$ Forensic Meteorology $•$ Direct-Access Real-Time Meteorological Data Base $\bullet$ Instrumentation Audits and Calibrations

568 Manzanita Ave, Suite 1 916-893-0308

Chico, CA 95926 FAX: $916-893-4517$
AIR SCIENCE CONSULTANTS, INC.

SKYWATCH WEATHER CENTER ${ }^{\circledR}$

RICHARD J. MANCINI, PRESIDENT

\section{Certified Consulting Meteorologist}

Forecasting Services for Industry, Government and News Media • Air Quality Modeling and Monitoring $\bullet$ Impact and Climatological Studies - Forensic Meteorology

347 Prestley Rd.

Bridgeville, PA 15017

412-221-6000

WEATHERDATA, INC.

MIKE SMITH, PRESIDENT

\section{Certified Consulting Meteorologists}

"When Decisions Depend on Weather" • Custom Weather Forecasts \& Storm Warnings $\bullet$ Meteorological Investigations for Legal Matters, Expert Testimony $\bullet$ Weather \& Climate Data Analysis

245 N. Waco, Ste. 310

316-265-9127

Wichita, KS 67202 FAX: 316-265-0371

\section{METEOROLOGICAL APPLICATIONS}

JAMES H. MEYER, PRESIDENT

\section{Certified Consulting Meteorologist}

Forensic and Applied Meteorology • Climatology \& Environmental Research $\bullet$ Meteorological \& Air Quality Instrumentation \& Monitoring - Fugitive Dust \& Cooling Tower Drift Studies • Expert Testimony

12926 Allerton Lane

Silver Spring, MD 20904

$301-384-1875$

ALEXIS B. LONG, PH.D. Certified Consulting Meteorologist

Weather Modification and Cloud Physics Research

144 Jasper Road

Bentleigh, Victoria 3204

Australia

(61)3-557-9938

\section{METEOROLOGICAL EVALUATION}

SERVICES, CO. INC. (MES)

PATRICK T. BRENNAN, PRESIDENT

\section{Certified Consulting Meteorologist}

Air-Quality Consulting • Expert Testimony •Industrial Meteorology • Nuclear Licensing Studies •Weather Investigations for Legal and Insurance Firms

165 Broadway

Amityville, NY 11701

516-691-3395

Certified Consulting Meteorologist: The certification program of the American Meteorological Society is aimed at fostering the establishment and maintenance of a high level of professional competency, and mature and ethical. counsel, in the field of consulting meteorology. Requirements of knowledge, experience, and character are determined by a five-person board. Objectives of the program and application procedures are described in full detail in the August 1995 Bulletin (pp. 1416-1420). 


\section{PROFESSIONAL DIRECTORY SPACE RESERVED FOR CERTIFIED CONSULTING METEOROLOGISTS}

\section{EARTH TECH, INC.}

DR. STEVEN R. HANNA DR. LLOYD L. SCHULMAN

JOSEPH S. SCIRE JOSEPH CHANG

\section{Certified Consulting Meteorologists}

Air Quality Modeling \& Development • Evaluation • Permitting • Data Analysis • Air Toxics • Acid Deposition $\bullet$ Ozone $\bullet$ Complex Terrain • Indoor Air Quality • Expert Testimony

196 Baker Avenue

$508-371-4200$

Concord, MA 01742

FAX: 508-371-4280

\section{SPENCER DUCKWORTH}

\section{Certified Consulting Meteorologist}

Weather-Related Marine Incident Evaluations • USCG Licensed,

Vessel Operator • Registered Engineer in California

3403 Tanager Ave

Davis, CA 95616

JWR, INC.

JACK W. REED, PRESIDENT

\section{Certified Consulting Meteorologist}

Practice Limited to Problems with Airblast Propagations \& Hot-Air Ballooning

5301 Central NE, Suite 220

Albuquerque, New Mexico 87108

$505-265-6550$

\section{ALPINE GEOPHYSICS}

T.W. TESCHE, PH.D.

\section{Certified Consulting Meteorologist}

Urban Airshed Modeling $\bullet$ Meteorological and Air Quality Modeling and Analysis $\bullet$ Emission Inventory Development $\bullet$ Aerometric Field

Program Design $\bullet$ Avalanche Forecasting

Post Office Box 17344

Covington, KY 41017

606-341-7502 FAX: 606-341-7508

\section{ENSERCH ENVIRONMENTAL CORPORATION LAURENCE A. LABRIE DOUGLAS J. FULLE Certified Consulting Meteorologists \\ Air Quality Modeling • Environmental Monitoring $\bullet$ Permitting $\bullet$ Expert Testimony • Environmental Software $\bullet$ Hazardous Waste Remedia- tion Services $\bullet$ Environmental Consulting \\ Lyndhurst, NJ 201-460-1900; Denver, CO 303-988-2202 Atlanta, GA 404-449-5800; Boston, MA 617-451-1201 Bellevue, WA 206-451-4600; and other offices nationwide Formerly the Environmental Division of Ebasco Services Incorporation}

\section{RAY HOXIT, PH.D.}

\section{Certified Consulting Meteorologist}

Detailed Weather Analyses • Forensic Meteorology and Expert Testimony • Climatological Studies • Specialized Weather Forecasts

P.O. Box 140

Lake Toxaway, NC 28747

704-884-7979

WILLIAM H. KLEIN, PH.D.

\section{Certified Consulting Meteorologist}

Applied Research $\bullet$ Expert Testimony $\bullet$ Climate Studies $\bullet$ Extended Forecasting • Systems Design • Technique Development • Statistical Meteorology $\bullet$ Synoptic Climatology

5225 Pooks Hill Road \#929 South

$301-897-5485$ Bethesda, MD 20814

$301-405-5412$

\section{WEATHERCOUNSEL}

FRED L. ZUCKERBERG

\section{Certified Consulting Meteorologist}

Consulting for Weather Sensitive Activities • Decision Strategies • Algorithm Design • Training Needs Defined and Satisfied • Research Studies

2 Glenwood Lane

East Hills, NY 1157-1411

$516-621-7551$

\section{RADIAN CORPORATION}

RICHARD R. BOYD

KAREN KOWALEWSKY HOWARD W. BALENTINE

\section{Certified Consulting Meteorologists}

Industrial Meteorology $\bullet$ Air Quality Modeling \& Monitoring $\bullet$ Remote Sensing $\bullet$ Environmental Impact $\bullet$ Risk Assessment $\bullet$ Emergency Response Planning $\bullet$ Hazardous Waste Management

Washington, DC Rochester, NY Sacramento, CA 703-713-1500 716-292-1870 916-362-5332

\section{WEATHERVISION}

(Formerly Gulf Coast Weather Service)

ANDY JOHNSON, OFFICE MANAGER

\section{Certified Consulting Meteorologist}

Expert Testimony • Complete Computerized Data Gathering • Video Graphics • Digital Nowcasting • Industrial Forecasting

P.O. Box 31113

Tampa, FL 33631-3113

TWX: 810-876-0618 813-287-0900 


\section{SPACE RESERVED FOR CERTIFIED CONSULTING METEOROLOGISTS}

DBS ASSOCIATES, INC./

IMPACT WEATHER, INC.

DAVID B. SPIEGLER, PRESIDENT

\section{Certified Consulting Meteorologist}

Weather, climate, and environmental products, services, studies, and information systems • Forensic meteorological services • Applied research in the atmospheric sciences

33 Concord Road, Billerica, MA 01821

Billerica, MA: 508-439-0095

Fax: 508-439-0106

Atlanta, GA: 404-442-9298

\section{CLIMATOLOGICAL CONSULTING CORPORATION}

\section{JEAN T. LEE}

\section{Certified Consulting Meteorologist}

Specializing in Forensic Meteorology

1914 Peter Pan Street

Norman, OK 73072

150 Shope Creek Road Asheville, NC 28805

405-321-8011

\section{ACCU WEATHER INC.}

ELLIOT ABRAMS, SENIOR VICE PRESIDENT

\section{Certified Consulting Meteorologist}

Meteorological consultants serving industry, government and the media • Forensic Services • Forecast Services • Expert Testimony - Complete Database • Applied Information Technologies

814-237-0309

619 W. College Avenue

State College, PA 16801

\section{TASC}

ARTHUR BASS, PH.D.

\section{Certified Consulting Meteorologist}

Leadership in Applied Information Technologies • Applied Research, Information Systems, Services, and Data Products for the Earth and Environmental Sciences

55 Walkers Brook Drive

$617-942-2000$ Reading, MA 01867

FAX: 617-942-7100

\section{ROBERT L. CARNAHAN}

\section{Certified Consulting Meteorologist}

Specializing in System Development, Meteorological User

Requirement Studies and Environmental Public Contact Programs.

13 Lancaster Road

Lewes, Delaware 19958
302-644-1809 FAX: 302-644-0803
AWS SCIENTIFIC, INC.

BRUCE H. BAILEY, PH.D., PRESIDENT

\section{Certified Consulting Meteorologist}

Atmospheric Field Research • Monitoring and Data Analysis • Applied Climatology •Instrumentation •Wind and Solar Energy • Engineering Meteorology $\bullet$ Modeling $\bullet$ Daylighting Simulation

3 Washington Square

518-869-5637

Albany, NY 12205 FAX: 518-869-5729

\section{GALSON CORPORATION}

\section{R. LELAND DAVIS, CEO}

CHRISTOPHER BEDFORD, MODELING/METEOROLOGY MGR.

JOHN W. WILSON, AIR QUALITY PROGRAM MANAGER

Certified Consulting Meteorologist

Air Toxics Mgmt. and pcAIR-1@ Data Mgmt. Sys. $\bullet$ Dispersion Modeling - Mesoscale Forecasting • Climatological Studies • AQ/Meteorological Monitoring • Source Testing $\bullet$ Permitting $\bullet$ Expert Testimony 6601 Kirkville Rd., East Syracuse, NY 13057 315-432-0506 Offices in Rochester, NY; New York, NY; Philadelphia, PA; and Albany, NY

FORENSIC METEOROLOGY ASSOCIATES, INC. WALTER A. LYONS, Ph. D., President

\section{Certified Consulting Meteorologist}

Nationwide Litigation Support/Expert Testimony $\bullet$ Lightning and Severe Storms • Local Microclimates • Air Quality • Climatology • Computer Graphics and Video Production • Time Lapse Camera Rentals

46050 Weld County Road 13

800-854-7219 Ft. Collins, CO 80524 e-mail: lyonsccm@csn.org

TRINITY CONSULTANTS, INC.

D. BRUCE TURNER, SENIOR CONSULTANT

\section{Certified Consulting Meteorologist}

Air Quality Consulting Dispersion Modeling Courses Worldwide • BREEZE ${ }^{\text {Im }}$ Dispersion Modeling PC Software • Expert Testimony

Atlanta • Baton Rouge $\bullet$ Chapel Hill $\bullet$

Dallas • Fort Collins • Houston • Kansas City

\section{CLIMATOLOGICAL CONSULTING CORPORATION STEVEN BUSINGER, PH.D.}

\section{Certified Consulting Meteorologist}

Specializing in Forensic Meteorology

Department of Meteorology

University of Hawaii at Manoa

2525 Correa Road

Honolulu, Hawaii 96822

150 Shope Creek Road (808) 956-8775 


\section{PROFESSIONAL DIRECTORY SPACE RESERVED FOR CERTIFIED CONSULTING METEOROLOGISTS}

PREDICTION AND EVALUATION SYSTEMS DR. ALLAN H. MURPHY DR. EDWARD S. EPSTEIN

\section{Certified Consulting Meteorologists}

Applications of probability and statistics • Model/forecast verification • Design/evaluation of forecasting experiments • Use/value of weather/ climate information $\cdot$ Short courses

3115 NW McKinley Drive

Corvallis, OR 97330-1139

8216 Inverness Hollow Terrace

Potomac, MD 20854-2627

503-754-7424

WAYNE R. SAND, Ph.D.

\section{Certified Consulting Meteorologist}

Specializing in Aviation Weather (Icing • Thunderstorms • Turbulence - Low Ceiling and Visibility • Windshear • Orographic Weather) •

Accident Weather Analysis • Expert Testimony

7020 Baseline Rd

Boulder, CO 80303-3141

Phone and FAX: 303-494-2741

\section{CLIMATOLOGICAL CONSULTING CORPORATION}

DOUGLAS A. STEWART, Ph. D.

\section{Certified Consulting Meteorologist}

Specializing in Forensic Meteorology

2393 Crawford Court

Lantana, FL 33462

150 Shope Creek Road

Phone and fax: 407-642-2981

Asheville, NC 28805-9795 704-298-4237

\section{GLOBAL ATMOSPHERIC RESPONSE \\ RUDOLF J. ENGELMANN, Ph.D}

\section{Certified Consulting Meteorologist}

Specializing in Environmental Sciences, Emergency Preparedness, and Health Physics

11701 Karen Drive

Potomac, MD 20854

Phone/Fax: 301-299-8784

\section{JIM CLARY \& ASSOCIATES \\ JAMES C. CLARY, JR. \\ President}

\section{Certified Consulting Meteorologist}

Air Quality Consulting, Training, Software, and Support

\section{Suite 660}

4835 LBJ Freeway

Dallas, TX 75244
Phone: 214-386-5995

Fax: 214-386-5994 E-mail:73113.3665@CompuServe.COM

\section{CLIMATOLOGICAL CONSULTING CORPORATION}

ROBERT N. SWANSON

Certified Consulting Meteorologist

Specializing in Forensic Meteorology

1216 Babel Lane

Concord, CA 94518

150 Shope Creek Road

(510) 676-2228

Asheville, NC 28805-9795

(704) 298-4237

\section{CLIMATOLOGICAL CONSULTING CORPORATION}

LEE E. BRANSCOME, Ph. D.

Certified Consulting Meteorologist

Specializing in Forensic Meteorology

7338 155th Place North

Palm Beach Gardens, FL 33418

407-744-4889
150 Shope Creek Road Asheville, NC 28805-9795 704-298-4237

\section{ATMOSPHERIC INFORMATION SYSTEMS \\ KIT K. WAGNER, Ph.D. \\ Certified Consulting Meteorologist}

Meteorological Analysis \& Modeling $\bullet$ Air Quality Analysis \& Modeling - Urban Airshed Modeling • Meteorological Research • Public Policy

- Meteorology \& Air Quality Training Programs

P.O. Box 721165

405-329-8707

Norman, OK 73070

FAX: 405-329-8717

\section{TRC ENVIRONMENTAL CORP.}

PIETRO A. CATIZONE

GEORGE W. WILKERSON

\section{Certified Consulting Meteorologists}

Environmental Consulting \& Research • Applied Meteorology • Air Quality and Meteorological Monitoring $\bullet$ Diffusion Modeling $\bullet$ Tracer

Studies • Air Toxics Monitoring • Expert Testimony

1-800-TRC-5601

Offices in major industrial centers throughout the United States

\section{ALTERNATIVE RESOURCES, INC.}

DAVID H. MINOTT, V.P. CYNTHIA L. BURKHART

\section{Certified Consulting Meteorologists}

Air Dispersion Modeling • Air Toxics • Risk Assessment • Environmental Permitting-Air, Water, Solid Waste • Serving Industry, Institutions, Public Works 


\section{SPACE RESERVED FOR CERTIFIED CONSULTING METEOROLOGISTS}

\section{EARTH TECH, INC.}

MICHAEL GUSKI

\section{Certified Consulting Meteorologists}

Air Quality Modeling \& Development • Evaluation • Permitting • Data Analysis • Air Toxics • Acid Deposition • Ozone • Complex Terrain • Indoor Air Quality • Expert Testimony

\section{AMS polo shirts}

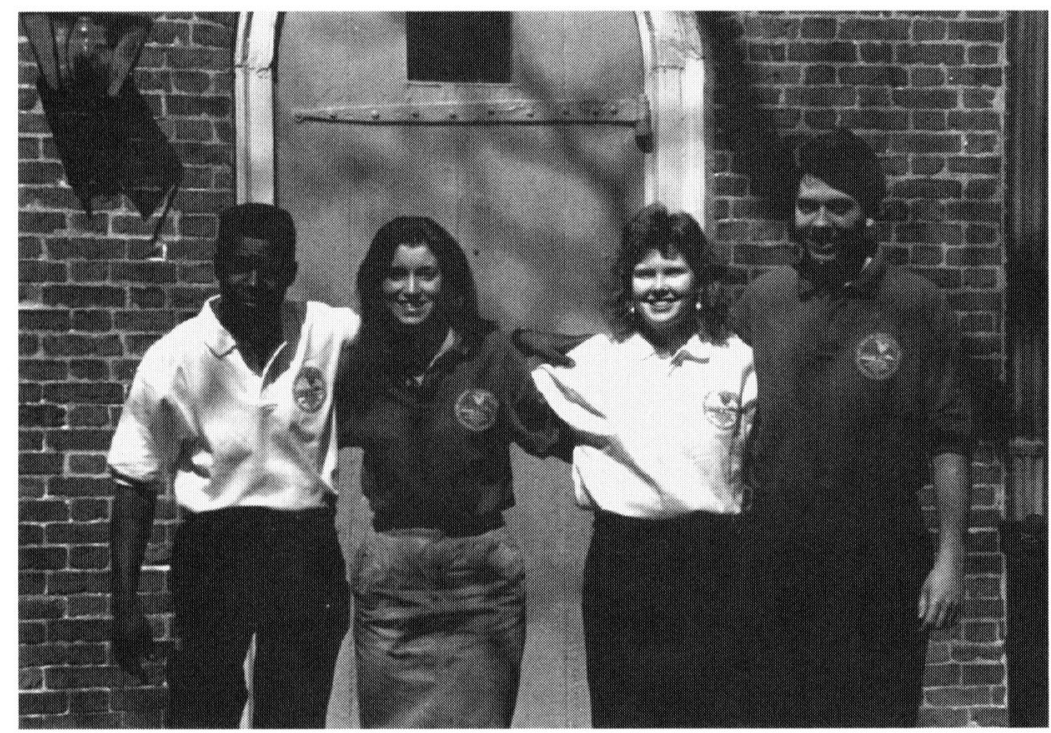

Made exclusively for the American Meteorological Society

The AMS polo shirt is made of sturdy $100 \%$ cotton, embroidered with the AMS seal. It comes in royal blue with a white seal or white with a blue seal, in $\mathrm{S}, \mathrm{M}, \mathrm{L}$, or XL. The price is $\$ 29.00$ per shirt, shipping and handling included (be sure to specify desired color and size). Please send prepaid orders to: Order Dept., American Meteorological Society, 45 Beacon St., Boston, MA 02108-3693. 


\section{SPACE RESERVED FOR MEMBERS}

\section{INTERNATIONAL WEATHER CORPORATION \\ WALTER F. ZELTMANN \\ Forensic Meteorology • Applied Climatology}

719 Bay Ridge Ave.

Brooklyn, NY 11220

$718-748-8066$

\section{WEATHERVISION \\ (formerly Gulf Coast Weather Service) WTVT Television Weather Services}

ROY LEEP, EXECUTIVE DIRECTOR

Complete Computerized Data Gathering • Video Graphics • Digital Nowcasting $\bullet$ Industrial Forecasting $\bullet P C$ Remote Accessing

P.O Box 31113

Tampa, FL 33631-3113

TWX 810-876-0618

813-287-0900

\section{ACCU WEATHER, INC.}

JOEL N. MYERS, PH.D., PRESIDENT

ELLIOT ABRAMS, M.S., CCM, SENIOR VICE PRESIDENT

JOSEPH P. SOBEL, PH.D., SENIOR VICE PRESIDENT

Weather Forecasting • AccuData Real-Time Database via PC, fax, Internet • Satellite, Radar and Graphics Images • Climatological and Forensic Services • 33 Years of Quality Service

619 W. College Ave. State College, PA 16801 Fax: 814-238-1339

E-mail: intemail@accuwx.com

\section{W. THORNTHWAITE ASSOCIATES}

WILLIAM J. SUPERIOR, PRESIDENT

Research \& Field Studies • Sensors \& Systems • Publications in Climatology

1725 Parvin Mill Road

Pittsgrove, NJ 08318

$609-358-2350$

\section{BRUCE F. WATSON}

\section{Consulting Meteorologist}

Research Specializing in Weather \& Climate of Minnesota \& Bordering States \& Provinces for Application to Agriculture,

Forensics, Engineering Studies, Environment, \& Marketing

\section{WEATHER SURVEYS}

CONRAD B. GOSSET, M.S., FORENSIC METEOROLOGIST

Specializing in Investigations for Law Firms \& Insurance Companies

P.O. Box 67

Cross River, NY 10518

$914-769-1350$

GEOMET TECHNOLOGIES, INC.

MARK J. STUNDER

Environmental Expert Systems, Artificial Intelligence • Air Pollution Analysis, Modeling \& Monitoring $\bullet$ Environmental Data Systems, Climatological Studies $\bullet$ Research and Operations

20251 Century Blvd.

Germantown, MD 20874

$301-428-9898$

\section{CENTRAL WEATHER SERVICE}

EARL S. FINCKLE, PRESIDENT

\section{Consulting Meteorologists}

Radio Weathercasting Experts \& Commodity Weather Specialist • Aviation, Business \& Industry, Government, Forensic Meteorology • Over a Quarter of a Century of Experience

1098 S. Milwaukee Ave.

$708-537-5920$

Wheeling, IL 60090 FAX 708-537-5926

\section{FLEETWEATHER, INC.}

\section{Since 1969}

\section{TORE H. JAKOBSEN, M.S., CO-OWNER}

Forecasts for Weather-Sensitive Industry $\bullet$ Past Weather Research \& Expert Testimony • Global Ship-Guidance \& Surveillance $\bullet$ Weathercasting • Automated Weather Databases \& Delivery Systems

The Fleetweather Building, 1966 Route 52 Hopewell Jct., NY 12533

FAX 914-226-1918 914-226-8300

Telex 673-3538

\section{METEOTEC INC. - An Alaska Science \& Technology Co.}

ROBERT L. BALDWIN, PRESIDENT

Environmental Science \& Engineering Consultants

Analysis $\bullet$ Systems $\bullet$ Sensing Communications $\bullet$ Automation $\bullet$ Wind \& Solar Energy • Air Quality $\bullet$ Noise $\bullet$ Modeling $\bullet$ Forensics • Management $\bullet$ Planning $\bullet R \& D$

P.O. Box 102915

Anchorage, AK 99510

907-562-2482 Telex 09026713 AKSCITECH AHG 


\section{SPACE RESERVED FOR MEMBERS}

NASH C. ROBERTS, JR., CONSULTANTS, INC.

\section{Consulting Meteorologists Since 1946}

General Industrial • Marine • Forensic • Air Quality • Agricultural • Commodities • Television

1040 N. Rampart St.

New Orleans, LA 70176

504-581-1688

FAX: 504-581-3165

\section{WEATHER CONSULTANTS OF CANADA CO. LTD (TELEWEATHER)}

MORRIS KESTIN, PRESIDENT

Long-Range Weather Outlooks \& Detailed Forecasts for Marketing Radio, TV, Movies, Publications, Commodities, Consultants to

Industry, Municipalities, Utilities, Forensic Meteorology since 1956

65 Marlborough Ave

Toronto, Ont., Canada M5R $1 \times 5$

416-968-7981

LIGHTNING LOCATION \& PROTECTION, INC.

Lightning Locating \& Warning Systems $\bullet$ Lightning \& Weather Radar Display Systems • Sensors for Automatic Meteorological Stations • Consulting on Lightning Hazards \& Lightning Protection.

2705 E. Medina Rd.

Suite 111

Tucson, AZ 85706

$602-741-2838$

\section{ESSCO - ENVIRONMENTAL SCIENCE AND SERVICES CORPORATION}

\section{WALTER G. HOYDYSH, PH.D., DIRECTOR}

Wind Tunnel Modeling • GEP Stack Height Demonstration • Dispersion Modeling $\bullet$ Ambient Air Quality Monitoring $\bullet$ Pedestrian Level Wind Studies $\bullet$ Wind Pressure \& Aeroelastic Studies
45-43 37th St.

Long Island City, NY 11101

718-786-3948 FAX 718-706-0391

\section{FRED W. DECKER, PH.D.}

Meteorologist Since 1937 (USWB, AAF, USAFR, Universities, Forensic). Physics Expert

Attorneys' Expert for Weather Reconstructions - Testimony and Cross-Examination Advice $\bullet$ Professor $\bullet$ Forecaster $\bullet$ Author of The Weather Workbook, Weather Analysis, Weather for Lawyers
COMPU-WEATHER, INC.

\section{Forecast and Research Specialists}

Weather Forecasting for Media, Industry, Government $\bullet$ Research Studies • Consultation • Forensic Meteorology • Excellent Client

References

1966 Route 52

Hopewell Junction, NY 12533

Phone: $914-227-8500$ Fax: $914-227-8400$

\section{YORK SERVICES CORPORATION}

Environmental Science \& Air Permits

Air Pollution Meteorology • Atmospheric Diffusion Modeling • Meteorological/Air Quality Monitoring Systems • Boundary-Layer Profiling

1 Research Drive

Stamford, CT 06906

Phone: 203-325-1371 Fax: 203-357-0166

\section{WSI CORPORATION}

Serves customers who depend on high quality weather information • value-added data • software packages • workstations • markets include media, aviation, industry, and government

4 Federal St

$508-670-5000$

Billerica, MA 0182

Telex 95-1184 FAX 508-670-5100

\section{EARTH ENVIRONMENT SERVICE}

STEVE NEWMAN, CHIEF METEOROLOGIST

Agriculture • Site Forecasts • Media • Graphics • Expert Testimony • Worldwide Forecasting

8200 St. Helena Rd.

707-539-1603

Santa Rosa, CA 95404

FAX707-538-5441

METEOROLOGICAL EVALUATION

SERVICES, CO. INC. (MES)

MAYNARD E. SMITH FRANK P. CASTELLI MARK L. KRAMER

\section{Consulting Meteorologists Since 1968}

Air-Qualtiy Modeling and Analysis • Forensic Meteorology • Monitoring and Data Analysis • Nuclear and Industrial Permitting Studies

165 Broadway

Amityville, NY 11701

$516-691-3395$ 
METRO WEATHER SERVICE, INC.

Servicing our clients for 15 years

Specialized Forecasts for the Media, Utilities \& Industry $\bullet$ Research \& Consultation $\bullet$ TV Weathercaster Training

507-535 Rockway Ave.

Suite \#3

Valley Stream, NY 11581

$516-568-8844$

\section{TECHNI-WEATHER CENTER}

WILLIAM JACQUEMIN, CHIEF METEOROLOGIST, PRESIDENT GREGORY CANTWELL, METEOROLOGIST, VICE PRESIDENT DOMINIC SCERBO, METEOROLOGIST

JEFFREY THOMAS, METEOROLOGIST

PROFESSIONAL SERVICES INCLUDING: Weather forecasting for Media, Utilities, Industry, Ski Areas, Government, Educational Svcs, Insurance/Lawyer Reports, Thunderstorm Research, Weatherphones

18 Woodside Ave.

Phone and Fax: 203-744-2064

Danbury, CT 06810-7123

\section{ALLIED WEATHER CONSULTANTS}

JOSEPH ALLEN, CHIEF FORECASTER

ERWIN K. KAUPER, CCM

Weather Forecasting Services for Utilities, Agriculture, Construction, and the Film Industry

P.O. Box 4584

Covina, CA 91723

818-967-1098

\section{ALDEN ELECTRONICS, INC.}

Distribution of global weather information - Lightning data $-N E X R A D$ - Satellite Communications Networks • Weather Information Data and Display

40 Washington Street

Westboro, MA 01581

$508-366-8851$

FAX 508-898-2427

TELEX 94-8404

\section{SECOR INTERNATIONAL INCORPORATED}

STEPHEN R. ANDERSEN

SIMS E. DUGGINS

PATRICK MCKEAN

Meteorological and Air Quality Measurements • Dispersion Modeling - Boundary Layer Studies • Data Analysis • Air Quality Permitting • Control Engineering $\bullet$ Emissions Measurements

4700 McMurray Dr., Suite 101

Ft. Collins, CO 80525

$303-226-4040$

FAX 303-226-4099
SURFACE SYSTEMS, INC.

JOE R. KELLEY, PRESIDENT/CEO

RICHARD C. CURTIS, DIRECTOR, WEATHER SERVICES

DAVID C. TRASK, CHIEF METEOROLOGIST

SPECIALIZING IN: Road and runway weather information systems • Operational forecasting and consulting for the utility and transportation industries

11612 Lilburn Park Road

314-569-1002

St. Louis, MO 63146

FAX 314-569-3567

\section{WEATHER TRADES, INC./ ROEMER WEATHER INC.}

JAMES ROEMER, PRESIDENT

Specializing in Commodity Trading \& Hedging Strategies Based on World Weather Conditions • Internet Services • Commodity Advisory Services • Trade Winds Newsletter

2411 Grand Ave., Suite 200 4208 Six Forks Rd., Suite 133 Raleigh, NC 27609

Des Moines, IA 50312-5309 Phone: $919-786-0844$

515-282-0233 Fax: 919-786-0948

E-mail: roemerwx@mercury.interpath.net

GLOBAL WEATHER DYNAMICS, INC.

Weather Services, Turnkey Systems

Worldwide Weather Services • Oceanographic Services • Forecasting $\bullet$ DataBanks $\bullet$ Applied Research $\bullet$ Surface and Satellite Communications $\bullet D E C$-, DC-, and PC-hosted Weather Message Switches

2400 Garden Road Monterey, CA 93940

408-649-4500 TELEX 171803 GWDI MNTY FAX 408-649-3288

\section{FOX WEATHER}

ALAN FOX, DIRECTOR

Satellite Analyses • Remote Sensing Studies • Site Forecasts • Extended Outlooks $\bullet$ Quantitative Precipitation Forecasts, Product Delivery via Internet

3924 W. Channel Islands Blvd

805-985-8743 Oxnard, CA 93035

FAX 805-985-3706

\section{COMMERCIAL WEATHER SERVICES ASSOCIATION}

JEFFREY C. SMITH, EXECUTIVE DIRECTOR

The national trade association for the private weather industry •

Brochure of professional weather forecasting and consulting companies and list of services is available by contacting CWSA.

655 Fifteenth St., NW, Suite 310

202-546-6993

Washington, D.C. 20005

FAX 202-546-2121 


\section{SPACE RESERVED FOR MEMBERS}

\section{R. M. YOUNG COMPANY}

Meteorological Instruments since 1964

Sensors to Measure: Wind Speed $\bullet$ Wind Direction $\bullet$ Peak Gusts • Temperature $\bullet$ Pressure $\bullet$ Relative Humidity $\bullet$ Precipitation

2801 Aero-Park Drive

Traverse City, Michigan 49686

616-946-3980 FAX 616-946-4772

\section{METEO CONSULT}

HARRY A. OTTEN, MANAGING DIRECTOR

WIM D. VAN DEN BERG, PH. D., DIRECTOR OF RESEARCH

Worldwide Forecasting with emphasis on Europe, Media, Marine (legally recognized in Dutch law), Agriculture, Software, Research and Consulting, and Lightning Data

P.O. Box 617

6700 AP Wageningen

The Netherlands

31837023300 FAX318370 23164

\section{SPECTRUM ENVIRONMENTAL SCIENCES, INC.}

THOMAS F. IACCARINO, MANAGING DIRECTOR

ROBERT W. JUBACH, MANAGING DIRECTOR

JAMES O. HOLIAN, MGR., METEOROLOGICAL PROGRAMS

Specializes in providing the following Air Quality Services:

Meteorological Data Analysis and Assessments, Meteorological \& Air Quality

Monitoring Programs, Audits, Atmospheric Field Programs, Forecasting, Air

Quality Permitting, Dispersion Modeling

Headquarters: $5735 \mathrm{C}$ Industry Lane, Ste. 10, Frederick, MD 21701

Phone: 301-620-1200; Fax: 301-620-4118

Frederick, MD

Braintree, MA

Springfield, MO

\section{GLOBAL ATMOSPHERICS, INC.}

THE NATIONAL LIGHTNING DETECTION NETWORK ${ }^{\mathrm{TM}}$

Worldwide lightning detection and location systems, ranging from wide area networks to local coverage sensors; Real-time, archive, and lightning verification data services

2705 E. Medina Road

Suite 111

Phone: $520-741-2838$

Tucson, AZ 85706

Fax: $520-741-2848$

Toll Free: $800-283-4557$

\section{WEATHER SUPPORT SERVICES}

BURTON L. SYLVERN, PRESIDENT

\section{Consulting Meteorologist}

Specialist since 1955

Weather Investigations for Law Firms and Insurance Agencies • Expert Testimony • Cli matological Studies

5690 Owls Nest Rd.

Tallahassee, FL 32308

904-668-0521

\section{AEROSPACE ENVIRONMENT}

ASSOCIATES (AEA)

PAUL JANOTA, PH.D., PRESIDENT

\section{Atmospheric Science and Technology}

Expertise in Applied Meteorology, Climatology, and Remote Sensing • Analyzing needs, developing business, and solving problems sensitive to the Aerospace Environment for Industrial and Government Clients

16 Hancock Street

Lexington, MA 02173

PHONE/FAX: 617-674-2981

\section{GOODMAN'S METEOROLOGICAL} SERVICES, INC.

MICHAEL GOODMAN, METEOROLOGIST and PRESIDENT

\section{Meteorological Instruments}

Sensors to monitor: Temperature, wind, relative humidity, precipitation, barometric pressure, and lightning. Individual units, remote monitoring units, and weather stations.

275 Eve Street

Manchester, NH 03104-1558

TOLL FREE 800-497-0571 FAX: 603-669-1990

WEATHERBANK, INC.

STEVEN A. ROOT, CCM

President/CEO

MICHAEL R. ROOT Executive Vice President/CFO

Operational Forecasting • Broadcast Services • Forensic Studies • Real-Time and Historical Weather Databases • Satellite Transmission Network - Weather/Air Quality Monitoring and Climatological Studies

\section{Over 23 Years of Providing Quality Service and Announcing Our New Location:}

1015 Waterwood Parkway, Suite J

$405-359-0773$ Edmond, Oklahoma 73034 FAX 405-341-0115

\section{MATRIX MANAGEMENT GROUP}

\section{General Management Consultants}

Meteorological Consultants to Governments and Industry • Project Management • Organization - Management Audits $\bullet$ Management Training • Policy Studies • Bridging the Gap Between Meteorology and Public Works

466 Colman Bldg.

Seattle, WA 98104

206-621-1977 202-842-4186

PC WEATHER PRODUCTS, INC.

GEORGE SAMBATARO, PRESIDENT/CHIEF METEOROLOGIST

Specializing in: Hurricane tracking/analysis software $\bullet$ Hurricane information systems • Global tropical cyclone tracking/analysis software $\bullet$ Hurricane historical analysis $\bullet$ Meteorological consultation 


\section{SIXTH CONIEREN(E ON \\ AVIATION WEATHER SUSTEMIS}

Aviolion Wecther Systems Overview Aviation Weather Forecasting Disseminationto

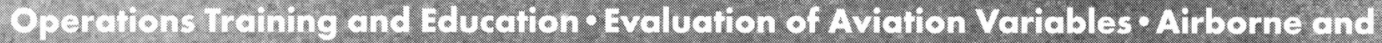

cround laing - Range and Aerospace Meteorology Ceiling and Visibility.

Integrofed Terminal Weother System-Speciol Air Weother Service Session?

Auromoted Observing Systems - Turbulence Low-Altitude Wind Shear

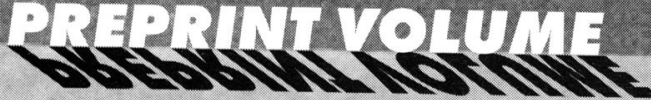

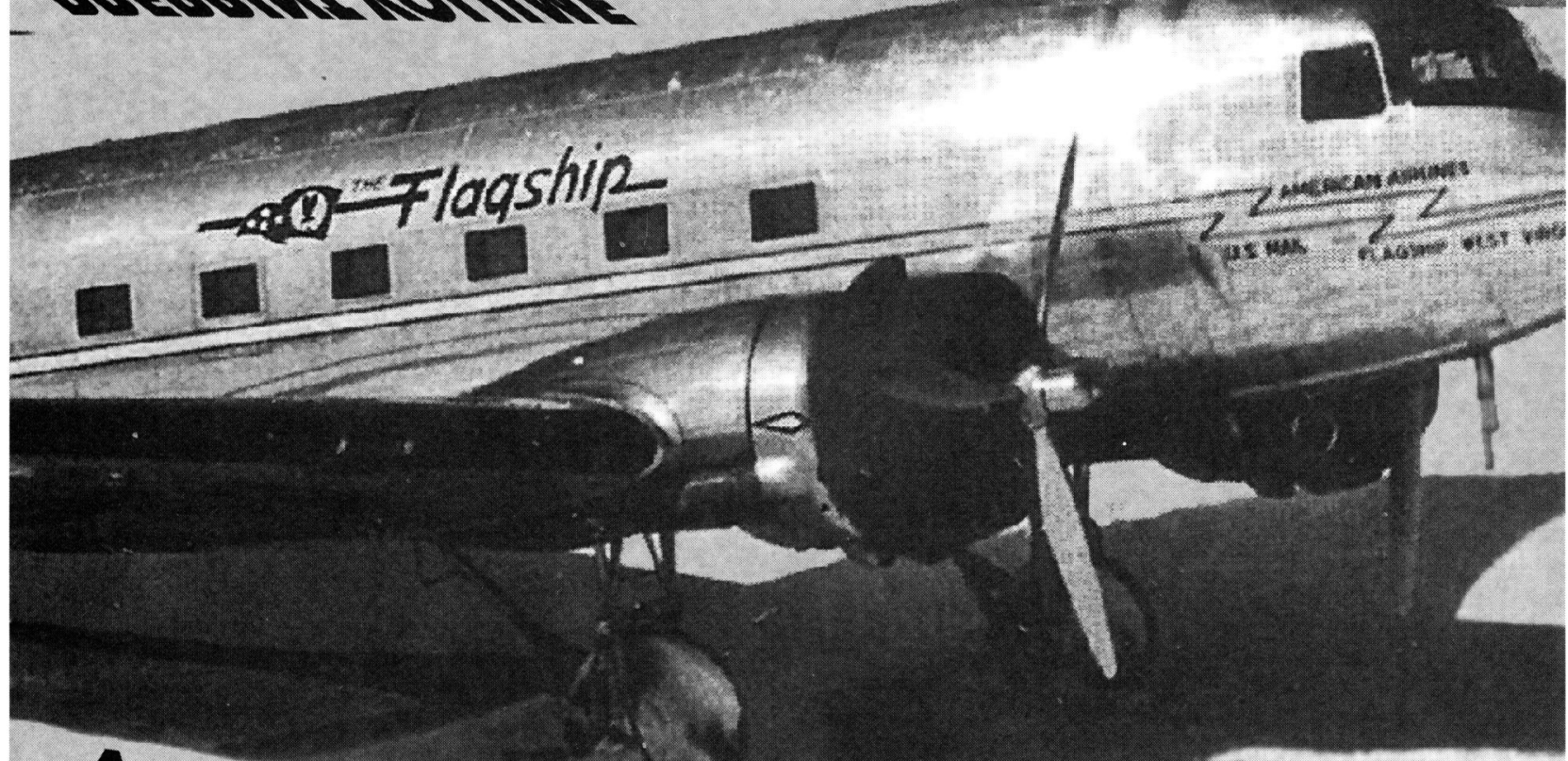

\section{American Meteorological Society}

\section{Dallas, Texas 15-20 January 1995}

C) 995 American Meteorological Society. Softbound, B\&W, 550 pp., $\$ 50.00$ list $\$ 35.00$ members (includes shipping and handling). Please send prepaid orders to: Order Department, American Meteorological Society, 45 Beacon St., Boston, MA 02108-3693. 


\title{
Monthly Weather Review
}

\author{
Vol. 124, No. 2, February 1996
}

\section{ARTICLES}

Diagnosis of an Amplifying and Decaying Baroclinic Wave Using Wind Profiler Data

Phillip L. Spencer, Frederick H. Carr, and Charles A. Doswell III

Investigating the Influence of Synoptic-Scale Monsoonal Winds and Mesoscale Circulations on Diurnal

Weather Patterns over Kenya Using a Mesoscale Numerical Model.

Joseph R. Mukabana and Roger A. Pielke

A Climatology of Anticyclones and Blocking for the Southern Hemisphere

MARK R. SinClair

Dynamics of Systematic Errors in the NMC Medium Range Forecast Model

Ming Cai, Jeffrey S. Whitaker, Randall M. Dole, and Kriste L. Paine

Simple Extensions of an NWP Model.

J. QIN AND H. M. van den DoOl

Long-Range Atmospheric Predictability Using Space-Time Principal Components

Robert Vautard, Carlos Pires, and Guy Plaut

Comparison of Thermodynamic Retrieval by the Adjoint Method with the Traditional Retrieval Method .........

AnoOK

An Objective Method for Inferring Sources of Model Error Seigfried Schubert and Yehui Chang

PICTURE OF THE MONTH

Langmuir Circulations in Rodeo Lagoon .... ANDREw J. SZERI

\section{Journal of Applied Meteorology}

\author{
Vol. 35, No. 2, February 1996
}

\section{ARTICLES}

Derived Product Imagery from GOES-8 …..... Christopher M. Hayden, Gary S. Wade, and Timothy J. Schmit A Comparison between Snow Cover Products Derived from Visible and Microwave Satellite Observations .... Alan Basist, Don Garrett, Ralph Ferraro, Norman Grody, and Kenneth Mitchell

Detection of Multilayer Cirrus Cloud Systems Using AVHRR Data: Verification Based on FIRE II IFO

Composite Measurements

Comparison of Modeled to Observed Global Irradiance

S. C. Ou, K. N. Liou, and B. A. Baum .. RichaRd F. DAVIS

Simulation of Surface Fluxes and Boundary Layer Development over the Pine Forest in HAPEX-MOBILHY

Optimal Estimation of Rain-Rate Profiles from Single-Frequency Radar Echoes Ziad S. Haddad, Eastwood Im, and Stephen L. Durden

Stochastic Filtering of Rain Profiles Using Radar, Surface-Referenced Radar, or Combined Radar-Radiometer

Measurements Ziad S. Haddad, Eastwood Im, Stephen L. Durden, and Scott Hensley

Biases Due to Gravity Waves in Wind Profiler Measurements of Winds

G. D. Nastrom and T. E. VanZandT

Coherent Observation Operators for Surface Data Assimilation with Application to Snow Depth

BERNARD URBAN

\section{NOTES AND CORRESPONDENCE}

A Possible Origin of Linear Depolarization Observed at Vertical Incidence in Rain
$153-162$

$163-177$

$178-191$

$192-201$

$202-213$

$214-228$

$229-242$

$243-257$

$258-270$

$271-277$ 


\title{
Journal of the Atmospheric Sciences
}

Vol. 53, No. 4, 15 February 1996

\section{ARTICLES}

Lee Vorticity Production by Large-Scale Tropical Mountain Ranges. Part I: Eastern North Pacific Tropical Cyclogenesis …....................................................................... Joel B. Mozer And JosePh A. ZeHnder

Lee Vorticity Production by Large-Scale Tropical Mountain Ranges. Part II: A Mechanism for the Production of African Waves ............................................................................... Joel B. Mozer And JosePh A. ZeHnder

The Potential Vorticity Budget of an Atmospheric General Circulation Model

J. N. Koshyk And N. A. McFarlane

Geostrophic versus Wave Eddy Viscosities in Atmospheric Models

.. Peter Bartello, Olivier Métais, and Marcel Lesieur

Natural Convection as a Heat Engine: A Theory for CaPE Nilton O. Rennó and Andrew P. Ingersoll

The Existence and Vertical Structure of Fast, Eastward-Moving Disturbances in the Equatorial Troposphere Ralph F. Milliff and Roland A. Madden

Turbulence Structure in Decoupled Marine Stratocumulus: A Case Study from the ASTEX Field Experiment Michael Tuernström and David P. Rogers

Radiative-Dynamical Consequences of Dry Tongues in the Tropical Troposphere

. Brian E. Mapes and Paquita Zuidema

The Life Cycle of a Simulated Marine Cyclone: Energetics and PV Diagnostics

G. Balasubramanian AND M. K. YaU

$521-538$

$539-549$

$550-563$

$564-571$

$572-585$

$586-597$

$598-619$

$620-638$

639-653

NOTES AND CORRESPONDENCE

On Calculating the Buoyancy of Cores in a Convective Boundary Layer

\section{Journal of Physical Oceanography}

\author{
Vol. 26, No. 2, February 1996
}

\section{ARTICLES}

Simulation of Mesoscale Variability in the Gulf of Mexico: Sensitivity Studies, Comparison with

Observations, and Trapped Wave Propagation

Convective Building of a Pycnocline: Laboratory Experiments David W. Pierce and Peter B. Rhines

The Cross-Slope Transport of Momentum by Internal Waves Generated by AlongSlope Currents over

Topography

Propagation of Gulf Stream Meanders between $74^{\circ}$ and $70^{\circ} \mathrm{W}$ .. S. A. Thorpe

Propagation and Growth of Gulf Stream Meanders Between $75^{\circ}$ and $45^{\circ} \mathrm{W}$.

Destruction of the Inertial Recirculation by the Annual Wind Migration
Tong Lee and Peter Cornillon Tong Lee and Peter Cornillon ZHENGYU LiU
$176-190$

191-204

205-224

225-241

$242-256$

\section{NOTES AND CORRESPONDENCE}

Evidence for Hydraulically Controlled Outflow of Brackish Water from Holandsfjord, Norway .....

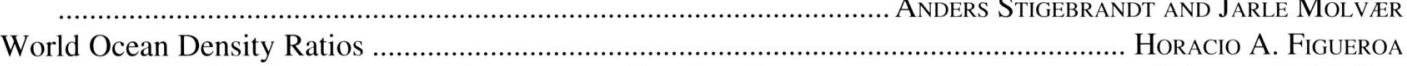

A Model for Surface Wave Propagation across a Shearing Current.

William D. McKeE

Asymptotic Stability of the Viscous-Plastic Sea Ice Rheology

R. M.S.M. SCHULKES

Comments on "Multiple Equilibrium States in Combined Thermal and Saline Circulation".......... JosEPH EGGER

Reply

Rui Xin Huang and James R. Luyten

$257-266$

$267-275$

276-278

279-283

284-285

286 


\section{Journal of Climate}

Vo 1. 9, No. 2, February 1996

\section{ARTICLES}

Interpretation of Interdecadal Trends in Northern Hemisphere Surface Air Temperature......

Evaluating the Potential Predictive Utility of Ensemble Forecasts

A Prognostic Cloud Water Parameterization for Global Climate Models

Anthony D. Del Genio, Mao-Sung Yao, William Kovari, and Kenneth K.-W. Lo

Global Observations of Upper-Tropospheric Water Vapor Derived from TOVS Radiance Data

Graeme L. Stephens, Darren L. Jackson, and Ian Wittmeyer

Climate Parameters from Satellite Spectral Measurements. Part I: Collocated AVHRR and HIRS/2

Observations of Spectral Greenhouse Parameter ... Richard A. Frey, S. A. Ackerman, and Brian J. Soden

Parameterizations of Marine Stratus Microphysics Based on In Situ Observations: Implications for GCMs ......

. I. Gultepe, G. A. IsaAc, W. R. Leaitch, and C. M. Banic

The Onset and Interannual Variability of the Asian Summer Monsoon in Relation to Land-Sea.....

Thermal Contrast Chengfeng Li and Michio Yanai

Low-Frequency Variability of Surface Air Temperature in a 1000-Year Integration of a Coupled .....

Atmosphere Model

Syukuro Manabe, AND Ronald J. STOUFFER

Low-Frequency Variability in the Arctic Atmosphere, Sea Ice, and Upper-Ocean Climate System

C. M. Bitz, David S. Battisti, R. E. Moritz, and J. A. Beesley

Snow Cover and Snow Mass Intercomparisons of General Circulation Models and Remotely Sensed Datasets . James Foster, Glen Liston, Randy Koster, Richard Essery, Helga Behr, Lydia Dumenil,

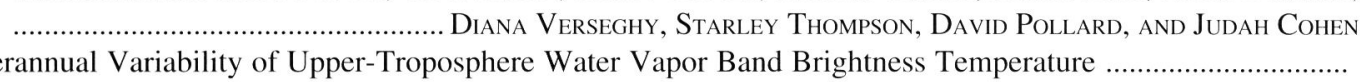

Interannual Variability of Upper-Troposphere Water Vapor Band Brightness Temperature ..............................

The Parameterization of Shortwave and Longwave Radiative Fluxes for Use in Zonally Averaged Climate Models

. Richard Bintanja

Association of Interannual and Interdecadal Variations of Global-Mean Temperature with Tropical Pacific

SST Appearing in a Model and Observations ............................................................ IN-SIK KANG

Northern Hemispheric Interannual Teleconnection Patterns and Their Changes Due to the Greenhouse Effect Xin-Zhong Liang, Wei-Chyung Wang, and Michael P. Dudek

\section{NOTES AND CORRESPONDENCE}

Recent Decrease of Sea Level Pressure in the Central Arctic 


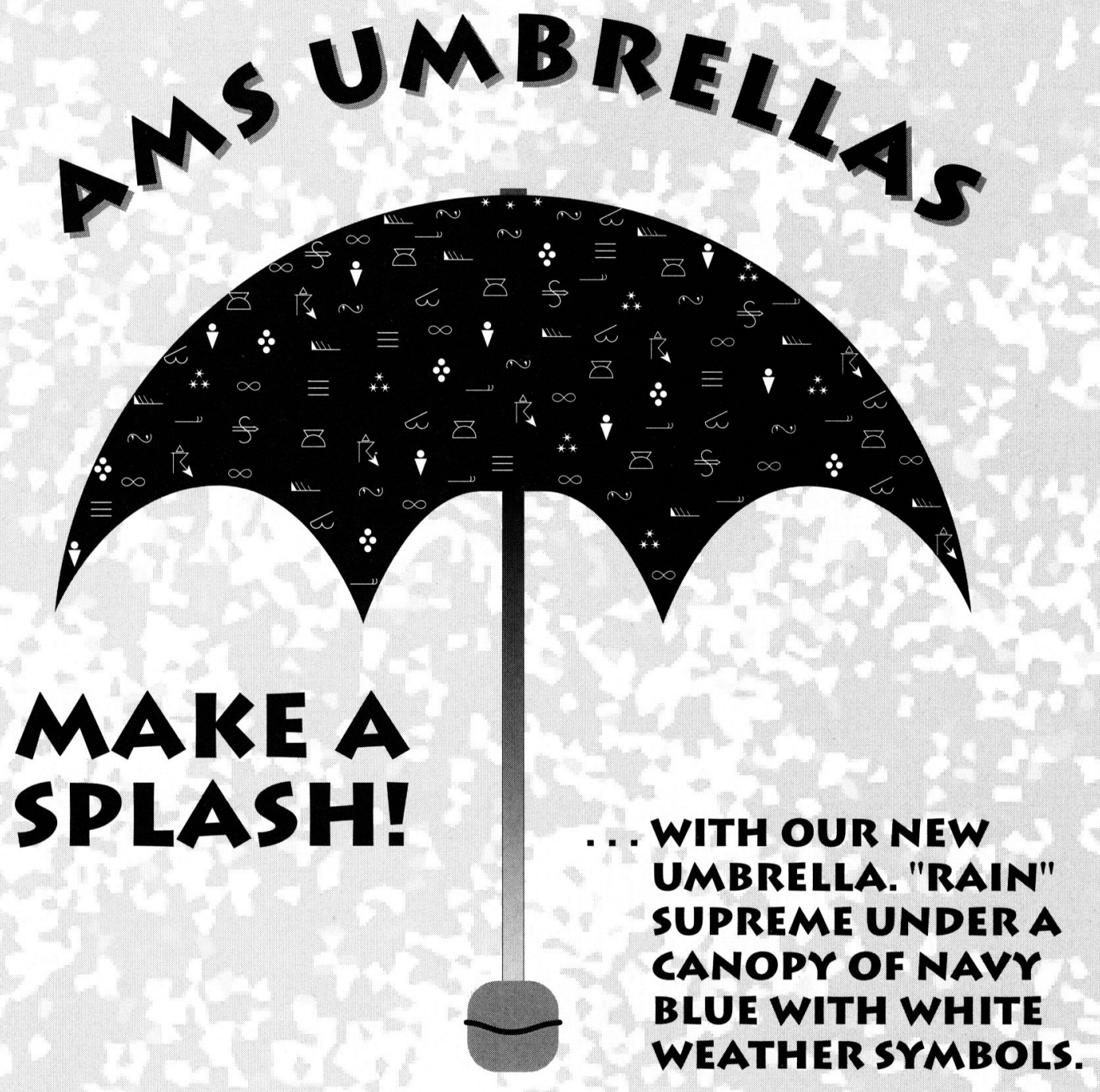

MADE EXCLUSIVELY FOR THE AMERICAN METEOROLOGICAL SOCIETY ... ORDER YOURS NOW!

\section{AMS Umbrellas - Only \$12 each!}

And that includes shipping and handling. Order with this form or by phone with MasterCard, Visa, or American Express at 617-227-2425, ext. 209/214/215/246.

Name:

Address:

City, State, ZIP:

Please send prepaid orders to: Order Department, American Meteorological Society, 45 Beacon St., Boston, MA 02108-3693.

MasterCard $\square$ Visa $\square$ American Express $\square$ Expiration Date:

Credit Card No. $\square \square \square \square \square \square \square \square \square \square \square \square \square \square \square \square$ 